\title{
An Optimized Slab-Symmetric Dielectric-Based Laser Accelerator Structure
}

\author{
J.B. Rosenzweig \\ UCLA Department of Physics and Astronomy \\ 405 Hilgard Ave., Los Angeles, CA 90095-1547 \\ P. V. Schoessow \\ Argonne National Laboratory \\ 9700 S. Cass Ave., Argonne, IL 60439
}

The submitted manuscript has been created by the University of Chicago as Operator of Argonne National Laboratory ("Argonne") under Contract No. W-31-109-ENG-38 with the U.S. Department of Energy. The U.S. Government retains for itself, and others acting on its behalf, a paid-up. nonexclusive. irrevocable worldwide license in said article to reproduce, prepare derivative works, distribute copies to the public, and perform publicly and display publicly, by or on behalf of the Government.

\begin{abstract}
A slab-symmetric, partially dielectric filled, laser excited structure which may be used to accelerate charged particles is analyzed theoretically and computationally. The fields associated with the accelerating mode are calculated, as are aspects of the resonant filling and impedance matching of the structure to the exciting laser. It is shown through computer simulation that the accelerating mode in this structure can be excited resonantly and with large quality factor $Q$. Practical aspects of implementing this structure as an accelerator are discussed.
\end{abstract}

The proposed use of lasers to accelerate charged particles has attracted much attention in recent years, spurred by the availability of increasingly large electromagnetic power densities from ever more sophisticated, yet cost-effective, laser sources. The proposed laser acceleration schemes range from mode conversion type devices, which the laser power is transformed into a different form of wave (e.g. plasma beat-wave accelerators[1]), to devices in which the laser is used to directly produce the accelerating fields. Direct laser accelerators can further be divided into two types of schemes - transverse-field dominated systems such as inverse Cerenkov accelerators[2], so-called vacuum accelerators, or other small-angle crossed laser beam devices[3,4], and systems in which the accelerating field profile is dominated by boundary conditions which are less than a vacuum wavelength away from the charged particle beam path [5]. The transverse-field dominated, or far-field, devices have the advantage of mitigating the fields on which induce breakdown on whatever structures are used to shape the field profile, but do so at a price - the longitudinal or accelerating fields are small compared to the transverse fields which may be experienced by the beam particles, leading to inefficient acceleration, and high sensitivity to asymmetries in the field profile. These asymmetries may lead to acceleration which is dependent on transverse position and concomitant strong transverse deflections of the beam particles.

On the other hand, in structures in which the field shaping boundary conditions are nearby, which are termed near-field structures, the material medium of the structure must support field levels a bit larger than the peak accelerating field experienced by the beam particles. For optical frequencies, the materials most resistant to field break down are dielectrics, which can support electric fields for short times (a few picoseconds), of a few GV/m[6], thus leading to a practical limit of about

\footnotetext{
- This work was supported by U.S. Dept. of Energy grants DE-FG03-93ER40796 and DE-FG0392ER40693, and the Alfred P. Sloan Foundation grant BR-3225.

Submitted to the proceedings of the 8th Workshop on Advanced Accelerator Concepts (AAC'98), Baltimore, MD, July 5-11, 1998.
} 


\section{DISCLAIMER}

This report was prepared as an account of work sponsored by an agency of the United States Government. Neither the United States Government nor any agency thereof, nor any of their employees, make any warranty, express or implied, or assumes any legal liability or responsibility for the accuracy, completeness, or usefulness of any information, apparatus, product, or process disclosed, or represents that its use would not infringe privately owned rights. Reference herein to any specific commercial product, process, or service by trade name, trademark, manufacturer, or otherwise does not necessarily constitute or imply its endorsement, recommendation, or favoring by the United States Government or any agency thereof. The views and opinions of authors expressed herein do not necessarily state or reflect those of the United States Government or any agency thereof. 


\section{DISCLAIMER}

Portions of this document may be illegible in electronic image products. Images are produced from the best available original document. 
$1 \mathrm{GeV} / \mathrm{m}$ acceleration rate in a dielectric-based device. This limit being recognized, near field structures present considerable advantages over far-field devices[5]: they can be made resonant, allowing much smaller peak power lasers to be used; symmetry in the fields can be enforced more straightforwardly; the periodicity of the field can be controlled to keep the beam particles synchronous with the accelerating wave. In addition, use of structures with slab, as opposed to cylindrical symmetry, gives the additional advantage of allowing a larger current to be loaded into the device without inducing excessive beam loading, as the accelerating beam can be spread out in the "wide" transverse dimension. Furthermore, it has been shown that use of a beam which is wide compared to the structure gap greatly suppresses the excitation of dipole modes in the device, thus enhancing the current which can be confidently accelerated before onset of the beam breakup instability[7].

Given these advantages, it was proposed in Ref. 5 that a structure based on a Fabry-Perot highly reflective mirror pair, loaded with a dielectric whose permittivity is modulated in the longitudinal dimension, be used as a near-field accelerator. The purpose of the permittivity modulation in the device was two-fold: first, to enforce the proper periodicity of the resonant fields, giving a field profile which is optimized for acceleration, and second, to modulate the phase of the incoming light to couple the electromagnetic power into the accelerating mode. Unfortunately, simulations of this geometry showed that it was difficult to couple into the accelerating mode in the structure, and that a strong non-accelerating component was always present in the mode. This paper presents a new, simplified geometry, in which the functions of shaping of the resonant fields in the structure, and coupling of the laser power to the structure, are split. It will be shown that the performance of this system is quite good, leading to fields which are strongly reminiscent of those found in standard radiofrequency linear accelerators.

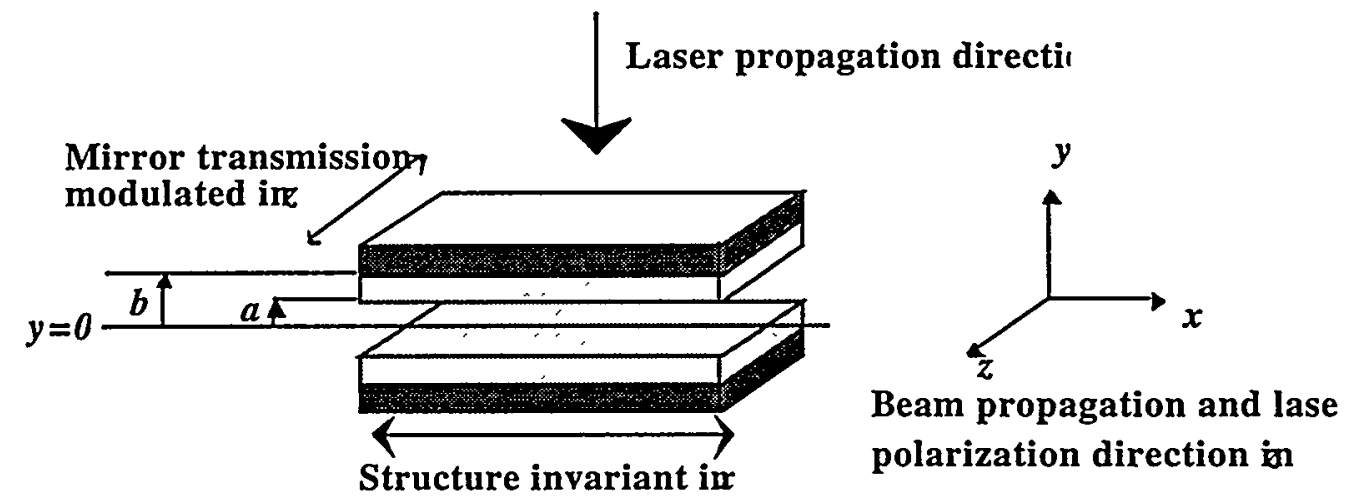

Metal $\square$ Dielectric

Figure 1. Geometry of slab-symmetric, laser-excited, dielectric-loaded resonant accelerator structure.

The structure geometry considered in this paper is shown in Figure 1. It consists of a pair of infinite (in $x$ and $z$ ) dielectric slabs of permittivity $\varepsilon$ and 
thickness $b-a$, separated by a gap of distance $2 a$. The slabs are clad at $|b|=b$ by metallic mirrors whose transmission coefficient $T$ is modulated periodically in $z$. While in practice the reflecting surfaces which bound the structure may be made of dielectric layers, the use of the metallic boundary conditions in our analysis allows simple evaluation of the eigenmodes we wish to excite without loss of generality. In this structure the separation of functions is now clear cut; the dielectric, with metallic boundary conditions at $|y|=b$, specifies the electromagnetic mode structure, while the modulation of the mirror transmission coefficient controls the coupling of the incoming laser power to the trapped mode, which instead of being Fabry-Perot-like, is now a standing wave pattern capable of accelerating charged particles.

The electromagnetic characteristics of the resonant modes of the structure are analyzed using the usual approximation of perfectly conducting metallic boundaries. In this limit, the fundamental mode for the structure can be simply deduced from previous work on travelling waves in such a device found in Ref. 7. Inside of the gap $(|y|<a)$, we have for the fundamental symmetric mode

$$
E_{z}=E_{0} \cos [k z] \cos [\omega t],
$$

where $E_{0}$ is the field amplitude and $\omega=k c$. This speed-of-light phase velocity condition is enforced by the periodicity of the modulation in the transmissivity, which thus must be chosen with to be the free-space wavelength of the exciting laser. Without preferential coupling to the speed-of-light phase velocity components (spatial harmonics), it should be noted that the structure is essentially invariant under displacement in $z$. The fields which obey this symmetry belong to what is termed in accelerator physics a "zero-mode", meaning a mode in which the phase advance per period is zero. This fundamental spatial harmonic of this mode is the familiar FabryPerot field pattern; the accelerating fields we wish to excite are actually at the first higher harmonic of the Floquet expansion of fields [5].

In the dielectric $(a<|y|<b)$ we have

$$
E_{z}=A E_{0} \cos [k z] \cos [\omega t] \sin [k \sqrt{\varepsilon-1}(y-b)],
$$

Application of the boundary conditions at $y=a$ allows the eigenvalue of this mode to be determined through the transcendental relation

$$
\cot (k \sqrt{\varepsilon-1}(b-a))=k a \frac{\sqrt{\varepsilon-1}}{\varepsilon},
$$

and the relative amplitude of the longitudinal electric field within the dielectric to be obtained,

$$
A=\csc [k \sqrt{\varepsilon-1}(b-a)] .
$$


For a gap much smaller than the free space wavelength $k a<<1$, the right hand side of Eq. 3 vanishes, and it is clear that $k \cong \pi / 2 \sqrt{\varepsilon-1}(b-a)$, and that $A \cong 1$. On the other hand, for a large gap $(k a \gg 1)$, the right hand side of Eq. 3 is large, $k \cong \pi / \sqrt{\varepsilon-1}(b-a)$, and $A \cong \sqrt{\varepsilon-1} / \varepsilon k a$. Thus for a large gap the field in the dielectric becomes much larger than that in the gap, and for a dielectric-breakdown limited situation the achievable accelerating field is strongly diminished. In this limit one can show that $E_{y}$ also becomes quite large near the dielectric boundary. It will also be shown below that large gap structures suffer from introduction of Fabry-Perot components of the mode. For all of these reasons, and despite the fact that it implies stringent limits on the allowable accepted beam emittance, operation with the small gap is strongly preferred.

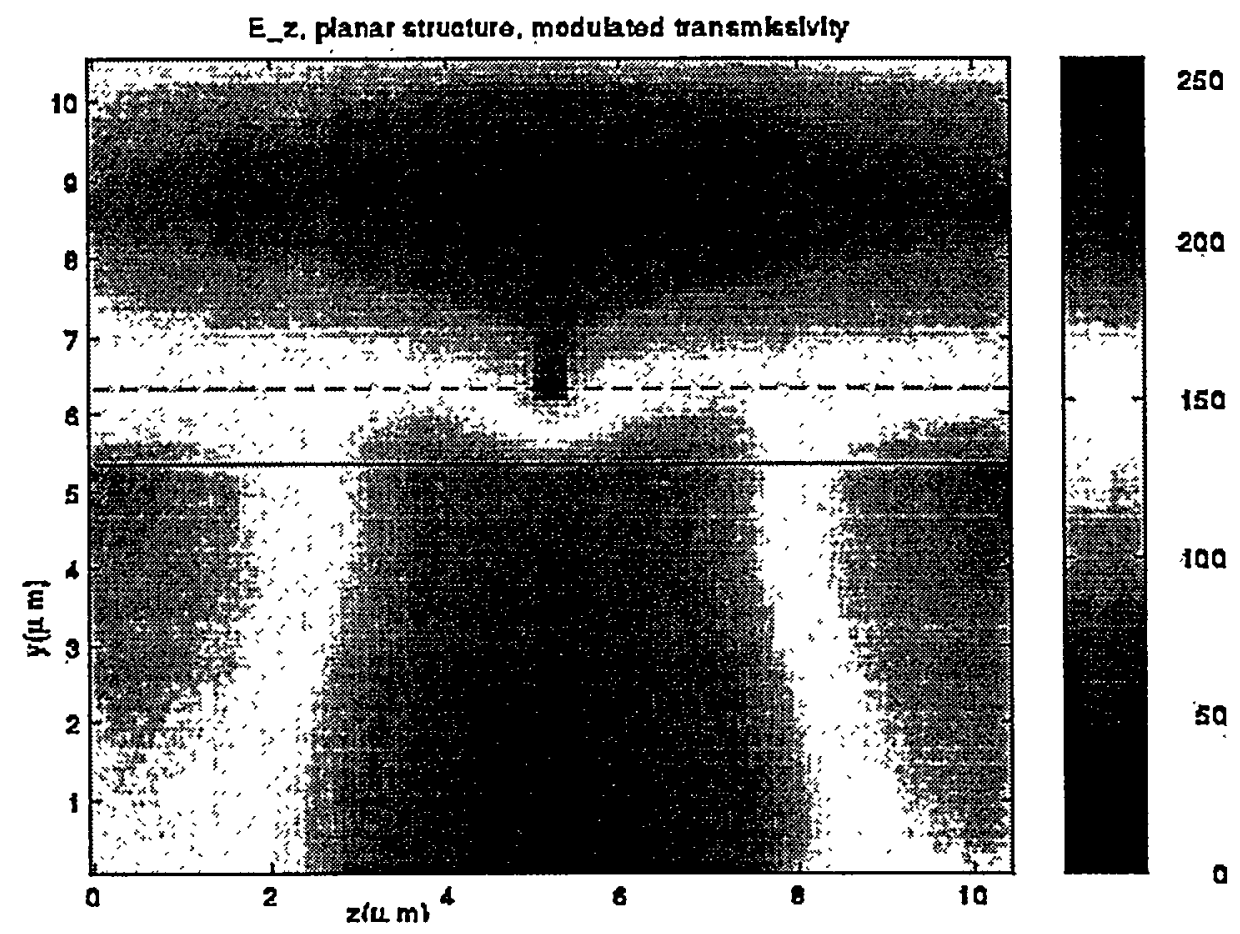

Figure 2. False-color contour map of $E_{\mathrm{z}}$ in dielectric-loaded structure with modulated transmission mirrors, calculated by electromagnetic solver. The maximum transmission occurs at $z=5.3 \_\mathrm{m}$. The laser wavelength is $\lambda=10.6 \mu \mathrm{m}$; the structure parameters are $a=5.3 \mu \mathrm{m}, b=6.27 \mu \mathrm{m}$ and $\varepsilon=3.47$.

The model we have chosen for the structure neglects dissipative losses in the dielectric, but will allow dissipation in mirrors, as this is a standard way of computationally constructing a partially transmissive boundary. Thus the quality factor $Q=\omega U / P$ is interpreted in the way familiar to designers of standing wave accelerators, with the power losses $P$ arising from ohmic losses in the cavity walls, as well as from fields radiated by the structure. Together, these effects combine to give an external $Q$ for the structure, with $1 / Q=1 / Q_{\text {ohmic }}+1 / Q_{\text {rad. }}$. Further, as we are interested in accelerating fields near the breakdown limit, we are not free to choose 
this external $Q$ to be arbitrarily large, even though a high $Q$ device would lessen the required input laser power. The smallness of the assumed $Q$ (in the range of 100 to $1000)$ in practice may imply that $Q_{\text {ohmic }} \gg Q_{\text {rad, }}$, which has implications for impedance matching of the structure to the input laser.

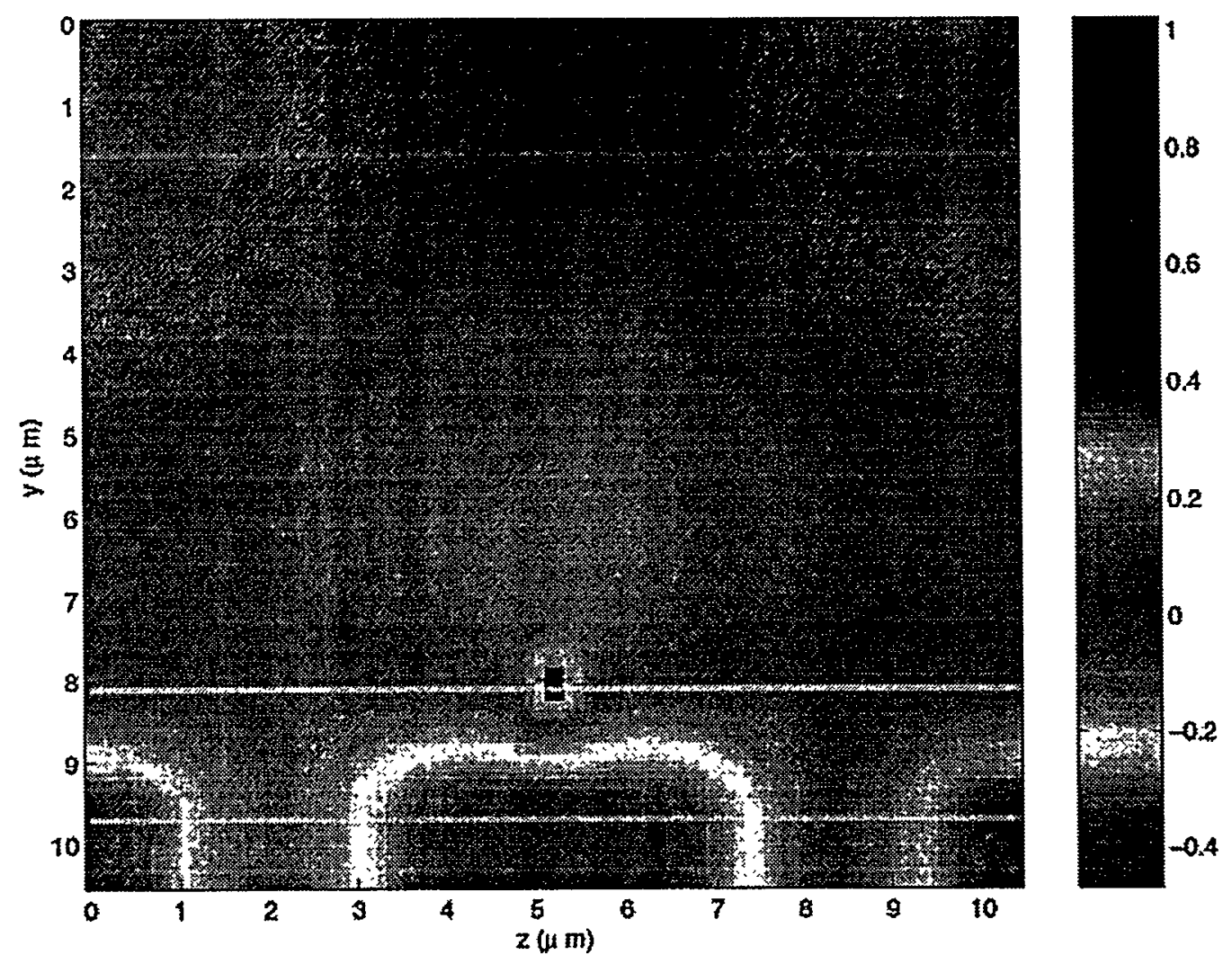

Figure 3. False-color contour map of $E_{z}$ in dielectric-loaded structure with modulated transmission mirrors, small gap case. The laser wavelength is $\lambda=10.6 \mu \mathrm{m}$; the structure parameters are $a=0.8 \mu \mathrm{m}, b=2.4 \mu \mathrm{m}$ and $\varepsilon=3.47$.

This structure has been studied by means of numerical simulations of the fields in a planar structure, performed using a custom 2-D (Cartesian) finite-difference time-domain electromagnetic solver code. Figure 2 shows a false-color contour map of $E_{\mathrm{z}}$ after many fill structure times for a case in which the exciting laser $(\lambda=10.6 \mu \mathrm{m})$ impinges on a structure with $a=5.3 \mu \mathrm{m}, b=6.27 \mu \mathrm{m}$ and $\varepsilon=2.35$. Because of the computational symmetry, the laser is implicitly injected into both top and bottow mirrors of the structure. The mirror transmission coefficient is varied sinusoidally with variation between zero $(z=0$ and $10.6 \mu \mathrm{m})$ and one percent $(z=5.3 \mu \mathrm{m})$. The gap size is large in this case, $k a=\pi$, and one can see that a pure accelerating mode, in which the phase fronts are normal to the beam (z) axis and $E_{z}$ is uniform in $y$, is not excited. The admixture of Fabry-Perot-like component, or spatial harmonic, in which $E_{z}$ is periodic in $y$ at the free-space wavelength $\lambda=2 \pi / k$, is non-negligible in this 
case, in fact it is nearly equal to the accelerating component. This is because with large $k a$ the fundamental Fabry-Perot mode in this structure is nearly degenerate in frequency with the fundamental accelerating mode, and thus the fields excited display some characteristics of both modes.

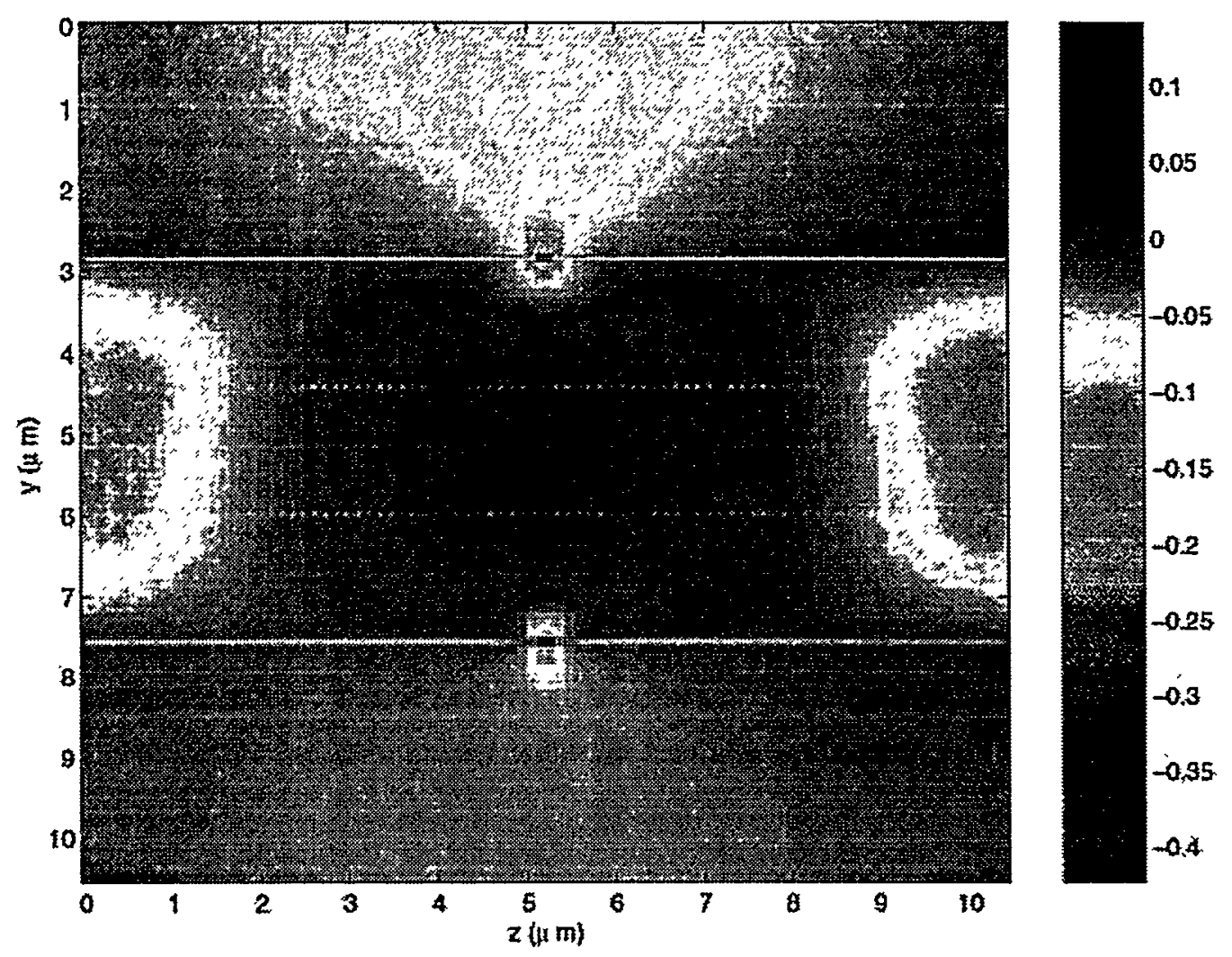

Figure 4. False-color contour map of $E_{z}$ in dielectric-loaded structure with modulated transmission mirrors, small gap case, without mirror symmetry enforced at $z=0$. The laser wavelength is $\lambda=10.6 \mu \mathrm{m}$; the structure parameters are $a=0.8 \mu \mathrm{m}, b=2.4 \mu \mathrm{m}$ and $\varepsilon=3.47$. Note that steady-state has not quite been reached, as the fields are still asymmetric within the structure.

This problem is overcome in the case of small $k a$, an example of which is displayed in Figure 3, where now $a=0.8 \mu \mathrm{m}, b=2.4 \mu \mathrm{m}$ and $\varepsilon=3.47$. The phase fronts $E_{z}$ are now much more uniform in $y$ in the gap, and the ratio of the accelerating to Fabry-Perot spatial harmonics is roughly $6: 1$. In addition, the field in the device is better enhanced compared to the laser field than the case shown in Fig. 2.

With the structure's accelerating mode well understood by theory and the computational model, we turn to discuss some aspects of the structure coupling to the drive laser. In practice, one will not necessarily drive both sides of the structure, but illuminate one side, and use the output radiation emitted from the opposite side as a diagnostic tool. This case is shown in Figure 4, which shows the same dielectricloaded structure as Fig. 3 without mirror symmetry enforced at $z=0$. In this case it can 
been seen that steady-state has not quite been reached, as the fields are still slightly asymmetric within the structure. In steady state with a lossless, impedance matched system, the field pattern should be symmetric with respect to the $z=0$ plane. The time dependence of the field amplitudes and stored (Figure 5) shows the approach to steady state typical of a high $Q$ resonator driven on its resonant frequency. In the case shown the $Q=80,000$ and the system has approached with characteristic fill time $\tau_{f}=Q / \omega=450 \mathrm{psec}$. This is in fact a higher $Q$ than one needs in practice, as large fill times will imply more problems with avalanche breakdown of the structure.

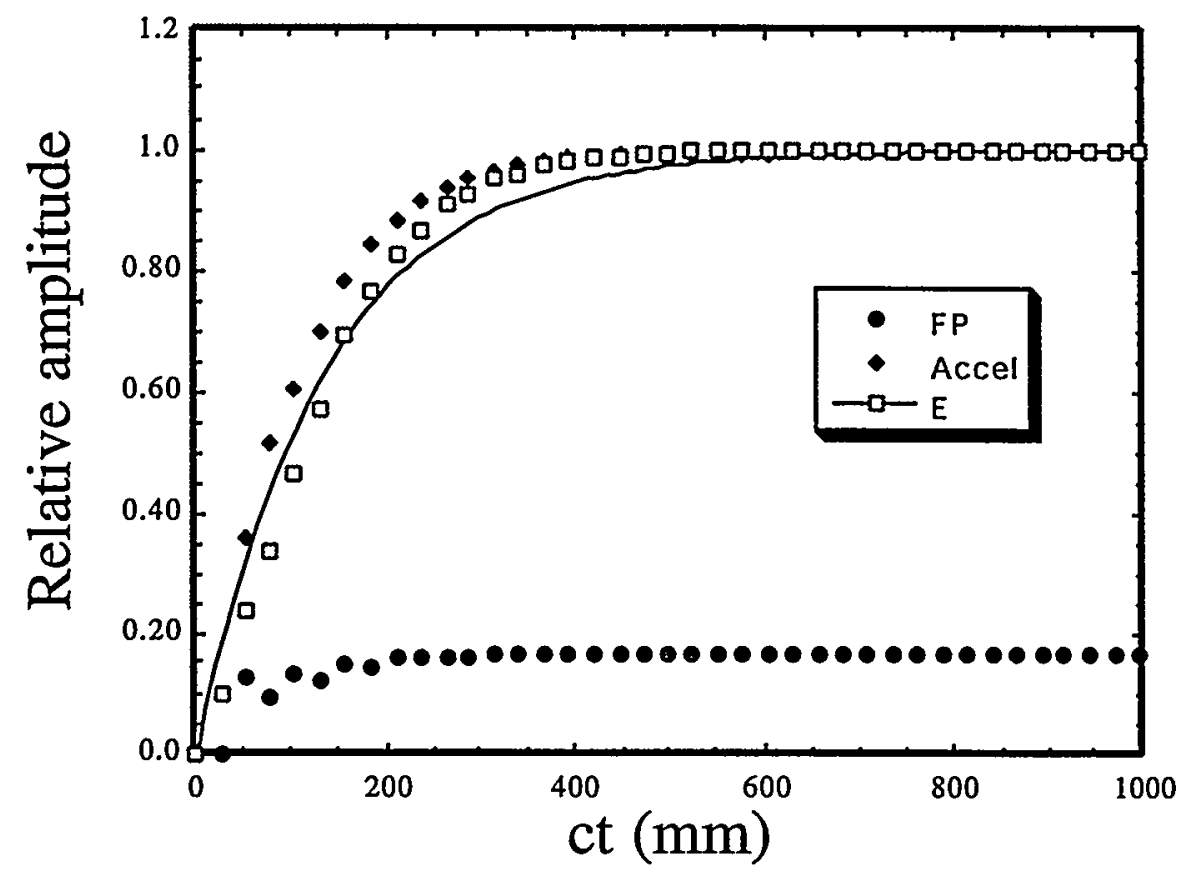

Figure 5. Time history of the accelerating (Accel), and Fabry-Perot (FP) components of the stored field in the structure for the case shown in Figure 4, with total stored energy (E).

In Figs. 3 and 4 , it can be seen that the coupling system, while driving the correct mode pattern, does so at the price of introducing a high-field region near the mirror's highest transmissivity (1\%) point. This is because, unlike a microwave cavity driven from a wave-guide, there is no region of free propagation linking the driving source and the resonant cavity. Thus the field must be large in the vicinity of the least poor transmissivity point. This effect in turn causes the introduction of higher spatial harmonics in the mode profile, which affect the field in the gap very little, as they exponentially attenuate in the vacuum region, but still serve to give a very high field region in the dielectric. This high field region undoubtedly would be the initiating point for structure breakdown.

In order to address this difficulty, it is sufficient to merely place small gaps in a uniform reflectance mirror at the periodicity of the free-space wavelength. This can be thought alternatively of as a simple deformation of the scheme shown in Figs. 3 and 4 , or as a reversion of the coupling system to the microwave cavity model., in 
which a slot is used to couple the wave-guide to the cavity. Promising preliminary simulations of this coupling scheme are now underway. They will be published in an upcoming work.

In conclusion, we have shown that it is possible to construct a simple, high $Q$ resonant device which can be efficiently coupled to an external laser source. This coupling scheme gives good selectivity for the desired accelerating mode component. Near-term future work will be centered on lowering the field values in the vicinity of the coupling slot. When this task is completed, we hope to begin practical engineering design work on such a system in preparation for experimental testing.

\section{REFERENCES}

1. C.E. Clayton, et al., Phys. Rev. Lett. 70, 37 (1993).

2. J.R. Fontana and R.H. Pantell, J. Appl. Phys. 54, 4285 (1983),

3. P. Sprangle, E. Esarey, A. Ting and G. Joyce, Appl. Phys. Lett. 53, 2146 (1988)?

4. Y.C. Huang, and R.L. Byer, Rev. Sci. Instrum. 69, 2629 (1998).

5. J.B. Rosenzweig, A. Murokh, and C. Pellegrini, Phys. Rev. Letters 74, 2467 (1995).

6. D. Du, et al., Appl. Phys. Lett. 64, 3073 (1994).

7. A. Tremaine, J. Rosenzweig, P. Schoessow, Physical Review E 56, 7204 (1997). 